\title{
A priori estimates for super-linear elliptic equation: the Neumann boundary value problem
}

\section{Estimations à priori pour l'équation elliptique super-linéaire : le problème de la valeur au bord de Neumann}

\author{
Abdellaziz Harrabi ${ }^{1,2}$, Belgacem Rahal ${ }^{3}$, and Abdelbaki Selmi ${ }^{4,5}$ \\ ${ }^{1}$ Department of Mathematics, Northern Border university, Arar, Saudi Arabia \\ ${ }^{2}$ Université de Kairouan, Département de Mathématiques, \\ Institut Supérieur des Mathématiques Appliquées et de l'Informatique \\ abdellaziz.harrabi@yahoo.fr \\ ${ }^{3}$ Institut Supérieur des Sciences Appliquées et de Technologie de Kairouan, \\ Avenue Beit El Hikma, 3100 Kairouan - Tunisia \\ rahhalbelgacem@gmail.com \\ ${ }^{4}$ Department of Mathematics, Northern Border university, Arar, Saudi Arabia \\ ${ }^{5}$ Université de Tunis, Département de Mathématiques, \\ Faculté des Sciences de Bizerte, Zarzouna, 7021 Bizerte, Tunisia \\ Abdelbaki.Selmi@fsb.rnu.tn
}

ABSTRACT. In this paper we study the nonexistence of finite Morse index solutions of the following Neumann boundary value problems

$$
(\text { Eq.H })\left\{\begin{array}{l}
-\Delta u=\left(u^{+}\right)^{p} \text { in } \mathbb{R}_{+}^{N}, \\
\frac{\partial u}{\partial x_{N}}=0 \quad \text { on } \partial \mathbb{R}_{+}^{N}, \\
u \in C^{2}\left(\overline{\mathbb{R}_{+}^{N}}\right) \text { and sign-changing, } \\
u^{+} \text {is bounded and } i(u)<\infty,
\end{array}\right.
$$

or

$$
\left(E q . H^{\prime}\right)\left\{\begin{array}{l}
-\Delta u=|u|^{p-1} u \text { in } \mathbb{R}_{+}^{N}, \\
\frac{\partial u}{\partial x_{N}}=0 \\
u \in C^{2}\left(\overline{\mathbb{R}_{+}^{N}}\right), \\
u \text { is bounded and } i(u)<\infty .
\end{array}\right.
$$

As a consequence, we establish the relevant Bahri-Lions's $L^{\infty}$-estimate [3] via the boundedness of Morse index of solutions to

$$
\begin{cases}-\Delta u=f(x, u) & \text { in } \Omega, \\ \frac{\partial u}{\partial \nu}=0 & \text { on } \partial \Omega,\end{cases}
$$

where $f$ has an asymptotical behavior at infinity which is not necessarily the same at $\pm \infty$. Our results complete previous Liouville type theorems and $L^{\infty}$-bounds via Morse index obtained in [3, 6, 13, 16, 12, 21].

2000 Mathematics Subject Classification. 35J60, 35J65, 58E05.

KEYWORDS. Morse index, Neumann boundary value problem, supercritical growth, Liouville-type problems, $L^{\infty}$-bounds.

\section{Introduction and main results}

The main purpose of this paper is to establish $L^{\infty}$-estimate for solutions of the following Neumann boundary value problem from the boundedness of their Morse indices

$$
\begin{cases}-\Delta u+u=f(x, u) & \text { in } \Omega \\ \frac{\partial u}{\partial \nu}=0 & \text { on } \partial \Omega\end{cases}
$$


where $\Omega \subset \mathbb{R}^{N}(N \geq 3)$ is a smooth bounded domain, $\frac{\partial}{\partial \nu}$ denotes the derivative with respect to the outward normal to $\partial \Omega$, and $f(x, t)$ with $f^{\prime}(x, t)=\frac{\partial f}{\partial t}$ are continuous on $\bar{\Omega} \times \mathbb{R}$. We focus on the class of nonlinearities having an asymptotical behavior at infinity which is not necessarily the same at $\pm \infty$. Precisely, we assume

$\left(H_{1}\right)$ There exist $p^{ \pm} \in\left(1, p_{c}(N)\right), p^{ \pm} \neq \frac{N+2}{N-2}$ with $p^{-} \leq p^{+}$such that

$$
\lim _{s \rightarrow \pm \infty} \frac{f^{\prime}(x, s)}{p^{ \pm}|s|^{p^{ \pm}-1}}=1 \text {, uniformly with respect to } x \in \bar{\Omega} .
$$

Moreover $p^{+} \in\left(\frac{N+2}{N-2}, p_{c}(N)\right)$ if $p^{-}<p^{+}$. 1

Here $p_{c}(N)$ is the so called Joseph-Lundgren exponent introduced in [17] (see also [6])

$$
p_{c}(N)= \begin{cases}\infty & \text { if } \quad N \leq 10 \\ \frac{(N-2)^{2}-4 N+8 \sqrt{N-1}}{(N-2)(N-10)} & \text { if } \quad N \geq 11\end{cases}
$$

We have not considered the subcritical case $1<p^{-}<p^{+}<\frac{N+2}{N-2}$ since it was completely achieved in [13]. So, we examine the cases $p^{+}=p^{-}$; or $p^{-}<p^{+}$and $p^{+} \in\left(\frac{N+2}{N-2}, p_{c}(N)\right)$ which are respectively discussed in [6] and [21] dealing with the following Dirichlet boundary value:

$$
\begin{cases}-\Delta u=f(x, u) & \text { in } \Omega, \\ u=0 & \text { on } \partial \Omega .\end{cases}
$$

Let-us first comment on some previous works using Morse index to provide Liouville theorems, $L^{\infty}$ bounds and existence results for problem (1.4). If $f(x, u):=|u|^{p-1} u+g(x, u)$ and $1<p<\frac{N}{N-2}$, where $g$ is not necessarily odd in $u$ and satisfying suitable subcritical conditions, Ramos-Tavares-Zou used the Morse index [20] to improve the celebrated multiplicity result of Bahri-Lions [2]. When the PalaisSmale; or the Cerami compactness conditions for the energy functional do not seem to follow readily, the proof of existence of solutions is essentially reduced to deriving $L^{\infty}$-bounds which in [19, 25] has been obtained where the authors adapted the approach of Bahri-Lions to prove the following theorem

Theorem 1.1. [3] Assume that $f$ satisfies

$\left(H_{2}\right)$ There exists $p \in\left(1, \frac{N+2}{N-2}\right)$ such that

$$
\lim _{|s| \rightarrow \infty} \frac{f^{\prime}(x, s)}{p|s|^{p-1}}=1, \text { uniformly with respect } x \in \bar{\Omega} .
$$

Let $\left(u_{n}\right) \in C^{2}\left(\bar{\Omega}\right.$ be a sequence of solutions of (1.4). Then $\left(u_{n}\right)$ is bounded in $L^{\infty}(\Omega)$ if and only if the sequence of their Morse indices $i\left(u_{n}\right)$ is bounded.

The key argument of their proof consists in showing the nonexistence of nontrivial finite Morse index solutions to the following equations

$$
-\Delta u=|u|^{p-1} u \text { in } \mathbb{R}^{N},
$$


and

$$
-\Delta u=|u|^{p-1} u \text { in } \mathbb{R}_{+}^{N} \text { and } u=0 \text { on } \partial \mathbb{R}_{+}^{N} .
$$

They investigated the subcritical case by using a spectral argument combined with Moser's iteration techniques and the Pohozaev identity (see also [10] for similar result for the polyharmonic problems). In a very interesting paper [6], Farina obtained a complete classification up the characteristic exponent $p_{c}(N)$, and therefore Theorem 1.1 holds true under $\left(H_{1}\right)$ with $p^{-}=p^{+}$. Also, the above $L^{\infty}$-bounds was extended for a large class of nonlinearities $f$ satisfying $\left(H_{1}\right)$ with $p^{ \pm} \in\left(1, \frac{N+2}{N-2}\right)$, where Harnack's inequality together with appropriate barrier functions allow to iterate a blow-up argument leading in particular to deal with finite Morse index solutions of

$$
-\Delta u=\left(u^{+}\right)^{p} \text { in } \mathbb{R}^{N},
$$

which reveals a new classification results [16]. Regarding the supercritical case, Rebhi improved the estimate obtained in [12] by extending the range of $p^{ \pm}$up to $p_{c}(N)$ [21]. Under some subcritical growth assumption weaker than $\left(\mathrm{H}_{2}\right)$, Yang succeeded to establish an explicit $L^{\infty}$-estimates [26] (see also [7, 9]). However, the general higher order case is harder to achieve since we need to carefully handle some local interior estimate, especially near the boundary (see [11] for the biharmonic and triharmonic cases). Point out that explicit estimate cannot be obtained for (1.1) since eventual extremal of $u$ could be appeared on $\partial \Omega$, so contrarily to [7, 11, 26], the Pohozaev identity fails to derive a local estimate. However, Bahri-Lions's $L^{\infty}$-bounds has been proved in the subcritical case with $p^{-}<p^{+}[13]$.

\section{Main Results}

We first classify finite Morse index solutions of the following Neumann boundary value problems on the half-space $\mathbb{R}_{+}^{N}=\left\{x=\left(x^{\prime}, x_{N}\right), x^{\prime} \in \mathbb{R}^{N-1}, x_{N}>0\right\}$ :

$$
(E q . H)\left\{\begin{array}{l}
-\Delta u=\left(u^{+}\right)^{p} \text { in } \mathbb{R}_{+}^{N}, \\
\frac{\partial u}{\partial x_{N}}=0 \quad \text { on } \partial \mathbb{R}_{+}^{N}, \\
u \in C^{2}\left(\overline{\mathbb{R}_{+}^{N}}\right) \text { and sign-changing, } \\
u^{+} \text {is bounded and } i(u)<\infty,
\end{array}\right.
$$

or

$$
\left(E q . H^{\prime}\right)\left\{\begin{array}{l}
-\Delta u=|u|^{p-1} u \text { in } \mathbb{R}_{+}^{N}, \\
\frac{\partial u}{\partial x_{N}}=0 \quad \text { on } \partial \mathbb{R}_{+}^{N}, \\
u \in C^{2}\left(\overline{\mathbb{R}_{+}^{N}}\right), \\
u \text { is bounded and } i(u)<\infty .
\end{array}\right.
$$

When $u$ is a solution of $(E q . H)$ or $\left(E q . H^{\prime}\right)$ and $\psi \in C_{c}^{1}\left(\mathbb{R}^{N}\right)$, contrarily to the Dirichlet boundary value problem 1.7 we have $u \psi \notin H_{0}^{1}\left(\mathbb{R}_{+}^{N}\right)$, and therefore $u \psi$ cannot be used as a test function if we compute the Morse index $i(u)$ in the space of test functions $H_{0}^{1}\left(\mathbb{R}_{+}^{N}\right)$; or $C_{0}^{1}\left(\mathbb{R}_{+}^{N}\right)$. Also, point out that the functional space of the associated Euler-Lagrange functional of problem (1.1) is $H^{1}(\Omega)$, then the adequate space of test functions of the associated quadratic form is also $H^{1}(\Omega)$. Therefore, we have here to consider $H^{1}\left(\mathbb{R}_{+}^{N}\right)$ (respectively $H^{1}(\Omega)$ ) as set of test functions. 
Definition 2.1. - The Morse index of a regular solution $u$ of (1.1) (denoted $i(u)$ ), is defined as the maximal dimension of all subspaces $X$ of $H^{1}(\Omega)$ such that

$$
Q_{u}(v):=\int_{\Omega}\left(|\nabla v|^{2}+v^{2}\right)-\int_{\Omega} f^{\prime}(x, u) v^{2}<0, \forall v \in X \backslash\{0\} .
$$

- $u$ is stable outside a compact set $\mathcal{K}$ of $\Omega$ if $Q_{u}(v) \geq 0$ for all $v \in C_{c}^{1}(\Omega \backslash \mathcal{K})$.

- If $u$ is a solution of (Eq.H) (respectively $\left.\left(E q . H^{\prime}\right)\right)$, we define

$$
\begin{aligned}
& Q_{u}(v)=\int_{\mathbb{R}_{+}^{N}}|\nabla v|^{2}-p \int_{\mathbb{R}_{+}^{N}}\left(u^{+}\right)^{p-1} v^{2}, \\
& \text { (respectively } \left.Q_{u}(v)=\int_{\mathbb{R}_{+}^{N}}|\nabla v|^{2}-p \int_{\mathbb{R}_{+}^{N}}|u|^{p-1} v^{2}, \forall v \in H^{1}\left(\mathbb{R}_{+}^{N}\right)\right) .
\end{aligned}
$$

\section{Remark 2.1.}

(1) Observe that if $u$ is a solution of $(E q . H)$ (respectively $\left(E q . H^{\prime}\right)$ ), then $Q_{u}(v)$ is well defined for all $v \in H^{1}\left(\mathbb{R}_{+}^{N}\right)$ as $u^{+}$(respectively $u$ ) is assumed a bounded function.

(2) Of course, if $\Omega=\mathbb{R}^{N}$ we use $C_{c}^{1}\left(\mathbb{R}^{N}\right)$ as a set of test functions.

(3) Clearly, any finite Morse index solution is stable outside a compact set.

(4) Any positive solution of $(E q . H)$ is a bounded solution of $\left(E q . H^{\prime}\right)$. If $u \leq 0$ is a solution of $(E q . H)$, then $u$ is an harmonic function and so $u=c$ with $c \leq 0$.

Problem 1.7 has been discussed in [6] where an odd extension was employed to provide the speed decay which is essential to derive the existence result. As far as we know, it is not proved in previous works, that the odd extended solution has finite Morse index (or stable outside a compact set). We used here the following even extension

$$
\bar{u}(x):= \begin{cases}u\left(x^{\prime}, x_{N}\right) & \text { if } x_{N} \geq 0 \\ u\left(x^{\prime},-x_{N}\right) & \text { if } x_{N}<0\end{cases}
$$

Define

$$
C_{c, s}^{1}\left(\mathbb{R}^{N}\right)=\left\{v \in C_{c}^{1}\left(\mathbb{R}^{N}\right) ; v\left(x^{\prime}, x_{N}\right)=v\left(x^{\prime},-x_{N}\right), \forall\left(x^{\prime}, x_{N}\right) \in \mathbb{R}^{N}\right\} \subset H^{1}\left(\mathbb{R}^{N}\right)
$$

and denote by $i_{s}(\bar{u})$ the Morse index of $\bar{u}$ related to the space of test functions $C_{c, s}^{1}\left(\mathbb{R}^{N}\right)$. Then, we have Lemma 2.1. Let $u$ be a finite Morse index solution of $(E q . H)$, or $\left(E q . H^{\prime}\right)$, then $i_{s}(\bar{u})$ is finite and $i_{s}(\bar{u}) \leq i(u)$.

Note that if $u$ is a solution of $(E q . H)$ (respectively $\left.\left(E q . H^{\prime}\right)\right)$, then $\bar{u} \in C^{2}\left(\mathbb{R}^{N}\right)$ and as a consequence of the above lemma $u$ verifies

$$
(E q . S)\left\{\begin{array}{l}
-\Delta \bar{u}=\left(\bar{u}^{+}\right)^{p} \text { in } \mathbb{R}^{N}, \\
\bar{u}\left(x^{\prime}, x_{N}\right)=\bar{u}\left(x^{\prime},-x_{N}\right), \\
\bar{u} \text { is bounded above, sign-changing and } i_{s}(\bar{u})<\infty,
\end{array}\right.
$$


respectively

$$
\left(E q . S^{\prime}\right)\left\{\begin{array}{l}
-\Delta \bar{u}=|\bar{u}|^{p-1} u \text { in } \mathbb{R}^{N}, \\
\bar{u}\left(x^{\prime}, x_{N}\right)=\bar{u}\left(x^{\prime},-x_{N}\right), \\
\bar{u} \text { is bounded and } i_{s}(\bar{u})<\infty .
\end{array}\right.
$$

The finiteness of the Morse index $i_{s}(\bar{u})$ will be sufficient to provide the following integral estimate. Following [6], we have

Proposition 2.1. Let $u \in C^{2}\left(\mathbb{R}^{N}\right)$ be a solution of (Eq.S') (respectively (Eq.S)). Then, there exists $R_{0}>0$ such that for every $\gamma \in\left[1, \gamma_{\max }\right)$, we have

$$
\int_{B_{R}}\left|\nabla\left(|u|^{\frac{\gamma-1}{2}} u\right)\right|^{2}+\int_{B_{R}}|u|^{p+\gamma} \leq C\left(1+R^{N-2 \frac{p+\gamma}{p-1}}\right), \forall R>2 R_{0},
$$

and

$$
\int_{\{R<|x|<4 R\}}|u|^{p+\gamma} \leq C R^{N-2 \frac{p+\gamma}{p-1}}, \forall R>R_{0},
$$

respectively

$$
\int_{B_{R}}\left|\nabla\left(\left(u^{+}\right)^{\frac{\gamma+1}{2}}\right)\right|^{2}+\int_{B_{R}}\left(u^{+}\right)^{p+\gamma} \leq C\left(1+R^{N-2 \frac{p+\gamma}{p-1}}\right), \forall R>2 R_{0} .
$$

Here $C=C\left(N, p, R_{0}, \gamma\right)$ is a positive constant independent of $R$.

If $\bar{u}$ is a solution of $\left(E q . S^{\prime}\right)$ and $\frac{N+2}{N-2}<p<p_{c}(N)$, inequality (2.3) will be useful to obtain the speed decay of $\bar{u}$ and its gradient at infinity. If $\bar{u}$ is a solution of $(E q . S)$ and $\frac{N+2}{N-2}<p<p_{c}(N)$ the monotonicity formula and Farina's approach fail to derive nonexistence results as in [18, 6, 14, 23] since from (2.4) we have only the integral estimate of $\bar{u}^{+}$. So, following [12, 21], we shall verify that $\bar{u}$ is spherically symmetric a round a point $x_{0}$, and the support of $\bar{u}^{+}$is a ball $B_{\rho_{0}}\left(x_{0}\right)$. Therefore, in view of the Pohozaev identity we derive that $\bar{u}^{+}=0$ as $\frac{N+2}{N-2}<p^{+}<p_{c}(N)$. Consequently $\bar{u}$ is a negative harmonic function, which implies that $\bar{u} \equiv c$ with $c \leq 0$ which is impossible since we assume that $u$ is sign-changing solution. Our Liouville theorem reads as follows

Theorem 2.1. 1. Assume that $\frac{N+2}{N-2}<p<p_{c}(N)$. Then (Eq.H) has no any sign-changing solution.

2. Assume that $1<p<p_{c}(N), p \neq \frac{N+2}{N-2}$. Then $\left(E q . H^{\prime}\right)$ has no any nontrivial solution.

Regarding the case of the whole space (where as mentioned in Remark 2.1 that the Morse index is computed in $C_{c}^{1}\left(\mathbb{R}^{N}\right)$ ), problem (1.8) is completely classified in the subcritical case in [15] and in the supercritical range in [21]. Precisely we have

Theorem 2.2. [6] 21] (1) Nontrivial finite Morse index solutions to (1.6) exist if and only if $p \geq$ $p_{c}(N), N \geq 11$, or $p=\frac{N+2}{N-2}, N \geq 3$.

(2) Problem (1.8) has no nontrivial finite Morse index solutions if $\frac{N+2}{N-2}<p<p_{c}(N)$.

According to Theorems 2.1 and 2.2, we can prove the following $L^{\infty}$-bounds via the boundedness of Morse index. 
Theorem 2.3. Suppose that $f$ satisfies $\left(H_{1}\right)$. Let $\left(u_{n}\right)$ be a sequence of solutions of (1.4). Then $\left(u_{n}\right)$ is bounded in $L^{\infty}(\Omega)$ if and only if the sequence of their Morse indices is bounded.

This paper is organized as follows. Section 3 is devoted to the proofs of Lemma 2.1, Proposition 2.1 and Theorem 2.1. In section 4 we develop the blow-up technique to prove Theorem 2.3.

\section{Proofs of Lemma2.1, Proposition 2.1] and Theorem 2.1.}

\subsection{Proof of Lemma2.1.}

Let $u$ be a solution of $(E q . H)^{2}$ and $\psi \in H_{s}^{1}\left(\mathbb{R}^{N}\right)$. From a simple change of variable we have

$$
\begin{aligned}
Q_{\bar{u}}(\psi) & =\int_{\mathbb{R}^{N}}|\nabla \psi|^{2}-p \int_{\mathbb{R}^{N}}\left(\bar{u}^{+}\right)^{p-1} \psi^{2} \\
& =2\left(\int_{\mathbb{R}_{+}^{N}}\left(|\nabla \psi|^{2}-p \int_{\mathbb{R}_{+}^{N}}\left(u^{+}\right)^{p-1} \psi^{2}\right), \forall \psi \in H_{s}^{1}\left(\mathbb{R}^{N}\right) .\right.
\end{aligned}
$$

Assume by contradiction that $i_{s}(\bar{u})>i(u)$. Denote $i_{s}(\bar{u})=i_{s}$, then there exist $\psi_{1}, \psi_{2}, \ldots, \psi_{i_{s}} \in H_{s}^{1}\left(\mathbb{R}^{N}\right)$ linearly independent such that

$$
Q_{\bar{u}}(\psi)<0, \forall \psi \in\left\langle\psi_{1}, \psi_{2}, \ldots, \psi_{i_{s}}\right\rangle \backslash\{0\}
$$

Obviously $\psi_{j \mid \mathbb{R}_{+}^{N}} \in H^{1}\left(\mathbb{R}_{+}^{N}\right)$, for all $j=1,2 \ldots, i_{s}$, and $\psi_{1 \mid \mathbb{R}_{+}^{N}}, \psi_{2 \mid \mathbb{R}_{+}^{N}}, \ldots, \psi_{i_{s} \mid \mathbb{R}_{+}^{N}}$ are also linearly independent. As $\psi_{j}\left(x^{\prime},-x_{N}\right)=\psi_{j}\left(x^{\prime}, x_{N}\right)$, and from (3.1) and (3.2), we derive that

$$
Q_{u}(\psi)<0, \forall \psi \in\left\langle\psi_{1 \mid \mathbb{R}_{+}^{N}}, \psi_{2 \mid \mathbb{R}_{+}^{N}}, \ldots, \psi_{i_{s \mid \mathbb{R}_{+}^{N}}}\right\rangle \backslash\{0\} .
$$

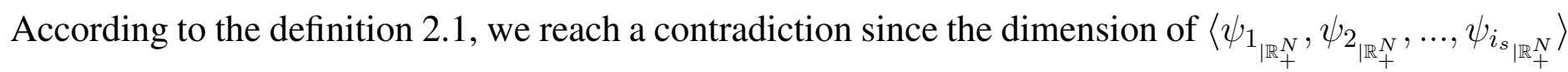
is equal to $i_{s}$ which is larger than $i(u)$.

\subsection{Proof of proposition 2.1.}

Denote by $B_{R}=\left\{x \in \mathbb{R}^{N},|x|<R\right\}$. The letter $C$ will be used throughout to denote a generic positive constant, which may vary from line to line and only depends on arguments inside the parentheses but does not depend on $R$. For the sake of simplification we denote confusedly $\bar{u}$ by $u$. From the finiteness of the Morse index $i_{s}(\bar{u})$ (where $\bar{u}$ is a solution of $(E q . S)$; or $\left(E q . S^{\prime}\right)$ ) we can find $R_{0}>0$ such that

$$
Q_{\bar{u}}(v) \geq 0, \forall v \in C_{c, s}^{1}\left(\mathbb{R}^{N}\right) \text { with } \operatorname{supp}(v) \subset \mathbb{R}^{N} \backslash B_{R_{0}} .
$$

Let $\psi \in C_{c}^{2}\left(\mathbb{R}^{N}\right)$ be a spherically symmetric cut-off function satisfying $\operatorname{supp}(\psi) \subset \mathbb{R}^{N} \backslash B_{R_{0}}$ and $0 \leq \psi \leq 1$. Set $m=\max \left(\frac{p+\gamma}{p-1}, 2\right)$.

2.The case of $u$ solution of $\left(E q \cdot H^{\prime}\right)$ is similar. 
Proof of (2.2). Clearly $|u|^{\frac{\gamma-1}{2}} u \psi^{m} \in C_{c, s}^{1}\left(\mathbb{R}^{N}\right)$ as $u\left(x^{\prime},-x_{N}\right)=u\left(x^{\prime}, x_{N}\right)$ and $\operatorname{supp}\left(|u|^{\frac{\gamma-1}{2}} u \psi^{m}\right) \subset$ $\mathbb{R}^{N} \backslash B_{R_{0}}$, for all $\gamma \geq 1$. Then from (3.3) we have

$$
p \int_{\mathbb{R}^{N}}|u|^{p+\gamma} \psi^{2 m} \leq \int_{\mathbb{R}^{N}}\left|\nabla\left(|u|^{\frac{\gamma-1}{2}} u \psi^{m}\right)\right|^{2}
$$

A simple calculation gives

$$
p \int_{\mathbb{R}^{N}}|u|^{p+\gamma} \psi^{2 m} \leq \int_{\mathbb{R}^{N}}|u|^{\gamma+1}\left(\left|\nabla \psi^{m}\right|^{2}-\frac{1}{2} \Delta\left(\psi^{2 m}\right)\right)+\int_{\mathbb{R}^{N}} \psi^{2 m}\left|\nabla\left(|u|^{\frac{\gamma-1}{2}} u\right)\right|^{2} .
$$

Now, multiply $\left(E q . S^{\prime}\right)$ by $|u|^{\gamma-1} u \psi^{2 m}$ and integrate by parts, we find

$$
\begin{aligned}
\int_{\mathbb{R}^{N}}|u|^{p+\gamma} \psi^{2 m} & =\gamma \int_{\mathbb{R}^{N}}|\nabla u|^{2}|u|^{\gamma-1} \psi^{2 m}+\frac{1}{\gamma+1} \int_{\mathbb{R}^{N}} \nabla\left(|u|^{\gamma+1}\right) \nabla\left(\psi^{2 m}\right) \\
& =\frac{4 \gamma}{(\gamma+1)^{2}} \int_{\mathbb{R}^{N}} \psi^{2 m}\left|\nabla\left(|u|^{\frac{\gamma-1}{2}} u\right)\right|^{2}-\frac{1}{\gamma+1} \int_{\mathbb{R}^{N}}|u|^{\gamma+1} \Delta\left(\psi^{2 m}\right) .
\end{aligned}
$$

Multiply the above inequality by $(1+\varepsilon) \frac{(\gamma+1)^{2}}{4 \gamma}, \varepsilon \in(0,1)$ and combine with (3.5), we derive

$$
\begin{aligned}
& \varepsilon \int_{\mathbb{R}^{N}} \psi^{2 m}\left|\nabla\left(|u|^{\frac{\gamma-1}{2}} u\right)\right|^{2}+\left(p-(1+\varepsilon) \frac{(\gamma+1)^{2}}{4 \gamma}\right) \int_{\mathbb{R}^{N}}|u|^{p+\gamma} \psi^{2 m} \\
& \leq C(p, \gamma, m) \int_{\mathbb{R}^{N}}|u|^{\gamma+1} \psi^{2 m-2}\left(|\nabla \psi|^{2}+|\Delta \psi|\right) .
\end{aligned}
$$

Using now Young's inequality with $q=\frac{p+\gamma}{\gamma+1}$ and $q^{\prime}=\frac{p+\gamma}{p-1}$ and taking into account that $(m-1) \frac{p+\gamma}{\gamma+1} \geq m$, we obtain

$$
\begin{aligned}
& \varepsilon \int_{\mathbb{R}^{N}} \psi^{2 m}\left|\nabla\left(|u|^{\frac{\gamma-1}{2}} u\right)\right|^{2}+\left(p-\varepsilon-(1+\varepsilon) \frac{(\gamma+1)^{2}}{4 \gamma}\right) \int_{\mathbb{R}^{N}}|u|^{p+\gamma} \psi^{2 m} \\
& \leq C(p, \gamma, m, \varepsilon) \int_{\mathbb{R}^{N}}\left(|\nabla \psi|^{2}+|\Delta \psi|\right)^{\frac{p+\gamma}{p-1}} .
\end{aligned}
$$

Observe that if $\gamma \in\left[1, \gamma_{\max }\right)$, then $p-\frac{(\gamma+1)^{2}}{4 \gamma}>0$, so we can choose $\varepsilon$ small enough such that $p-\varepsilon-$ $(1+\varepsilon) \frac{(\gamma+1)^{2}}{4 \gamma}>0$. Thus, we obtain

$$
\int_{\mathbb{R}^{N}} \psi^{2 m}\left|\nabla\left(|u|^{\frac{\gamma-1}{2}} u\right)\right|^{2}+\int_{\mathbb{R}^{N}}|u|^{p+\gamma} \psi^{2 m} \leq C(p, \gamma, m) \int_{\mathbb{R}^{N}}\left(|\nabla \psi|^{2}+|\Delta \psi|\right)^{\frac{p+\gamma}{p-1}}
$$

For $R>2 R_{0}$, define $\psi_{R} \in C_{c}^{2}\left(\mathbb{R}^{N}\right), 0 \leq \psi_{R} \leq 1$ such that

$$
\begin{cases}\psi_{R} \equiv 1 & \text { if } 2 R_{0}<|x|<R \\ \psi_{R} \equiv 0 & \text { if }|x|<R_{0} \text { or }|x|>2 R \\ \left|\nabla \psi_{R}\right| \leq \frac{C}{R},\left|\Delta \psi_{R}\right| \leq \frac{C}{R^{2}} & \text { if } R<|x|<2 R\end{cases}
$$


Consequently substitute $\psi$ by $\psi_{R}$ in inequality (3.6), we obtain

$$
\int_{B_{R}}\left|\nabla\left(|u|^{\frac{\gamma-1}{2}} u\right)\right|^{2}+\int_{B_{R}}|u|^{p+\gamma} \leq C\left(N, R_{0}, p, m, \gamma\right)\left(1+R^{N-2 \frac{p+\gamma}{p-1}}\right) .
$$

So, inequality (2.2) follows.

Proof of (2.3). For $R>R_{0}$, define $\psi_{R} \in C_{c}^{2}\left(\mathbb{R}^{N}\right), 0 \leq \psi_{R} \leq 1$ such that

$$
\begin{cases}\psi_{R} \equiv 1 & \text { if } R<|x|<4 R \\ \psi_{R} \equiv 0 & \text { if }|x| \leq \frac{R}{2} \text { or }|x| \geq 5 R, \\ \left|\nabla \psi_{R}\right| \leq \frac{C}{R},\left|\Delta \psi_{R}\right| \leq \frac{C}{R^{2}} & \text { if } 4 R<|x|<5 R\end{cases}
$$

So, $\operatorname{supp}(\psi) \subset \mathbb{R}^{N} \backslash B_{R_{0}}$. Substitute $\psi$ by $\psi_{R}$ in inequality (3.6), we derive

$$
\int_{\{R<|x|<4 R\}}|u|^{p+\gamma} \leq C(N, p, m, \gamma) R^{N-2 \frac{p+\gamma}{p-1}} .
$$

Hence, inequality (2.3) is well proved.

Proof of (2.4). We use here $\left(u^{+}\right)^{\gamma} \psi^{m} \in C_{c, s}^{1}\left(\mathbb{R}^{N}\right)$ as a test function. The proof is similar to the one of inequality (2.2). So we omit the proof here.

\subsection{Proof of Theorem 2.1.}

Proof of (1.) If $\frac{N+2}{N-2}<p<p_{c}(N)$, then $\gamma_{1}=(p-1) \frac{N}{2}-p \in\left[1, \gamma_{\max }\right)$ (see [6]) and from (2.4) $u=\bar{u}$ satisfies

$$
\int_{\mathbb{R}^{N}}\left(u^{+}\right)^{p+\gamma_{1}}<\infty .
$$

Recall first that if $f \in L^{q}\left(\mathbb{R}^{N}\right), q>1$ and $g \in L^{q^{\prime}}\left(\mathbb{R}^{N}\right)$ where $q^{\prime}$ is the conjugate exponent of $q$, then $f * g \rightarrow 0$ as $|x| \rightarrow \infty$. Define

$$
W(x):=C_{N} \int_{\mathbb{R}^{N}} \frac{\left(u^{+}\right)^{p}(y)}{|x-y|^{N-2}} d y, \text { where } C_{N} \text { is a positive constant . }
$$

Thus, we have

$$
\begin{aligned}
W(x) & =C_{N} \int_{\mathbb{R}^{N}} \frac{\left(u^{+}\right)^{p}(x-y)}{|y|^{N-2}} d y \\
& \leq C_{N} \int_{B_{1}} \frac{\left(u^{+}\right)^{p}(x-y)}{|y|^{N-2}} d y+C_{N}^{\prime} \int_{\mathbb{R}^{N}} \frac{\left(u^{+}\right)^{p}(x-y)}{(1+|y|)^{N-2}} d y .
\end{aligned}
$$

Set

$$
W_{1}=C_{N}\left(u^{+}\right)^{p} * \chi_{B_{1}}|y|^{2-N} \text { and } W_{2}=C_{N}^{\prime}\left(u^{+}\right)^{p} *(1+|y|)^{2-N},
$$


then $0 \leq W \leq W_{1}+W_{2}$. As $u^{+} \in L^{p+\gamma_{1}}\left(\mathbb{R}^{N}\right)$ and $u^{+}$is bounded then $u^{+} \in L^{q}\left(\mathbb{R}^{N}\right)$ for all $q \geq p+\gamma_{1}$. So choose $q$ large enough such that $q^{\prime}(N-2) \leq N$ which implies that $\chi_{B_{1}}|y|^{2-N} \in L^{q^{\prime}}\left(\mathbb{R}^{N}\right)$ and therefore $W_{1} \rightarrow 0$ as $|x| \rightarrow \infty$. Observe now that $\left(u^{+}\right)^{p} \in L^{\frac{p+\gamma_{1}}{p}}\left(\mathbb{R}^{N}\right)$ and $\frac{p+\gamma_{1}}{\gamma_{1}}$ which is the conjugate of $\frac{p+\gamma_{1}}{p}$ satisfying $(N-2) \frac{p+\gamma_{1}}{\gamma_{1}}>N$ as $\gamma_{1}=(p-1) \frac{N}{2}-p$. Hence, $(1+|y|)^{2-N} \in L^{\frac{p+\gamma_{1}}{\gamma_{1}}}\left(\mathbb{R}^{N}\right)$ and then $W_{2} \rightarrow 0$ as $|x| \rightarrow \infty$. Consequently $W$ is well defined and $W \rightarrow 0$ as $|x| \rightarrow \infty$. Also, as $u^{+} \in L^{p+\gamma_{1}}\left(\mathbb{R}^{N}\right)$ and $u$ is bounded above then from $L^{p} \rightarrow L^{q}$ theory we derive that $W \in C^{2}\left(\mathbb{R}^{N}\right)$ and satisfies $-\Delta W=\left(u^{+}\right)^{p^{+}}$in $\mathbb{R}^{N}$. Therefore, $\Delta(u-W)=0$ in $\mathbb{R}^{N}$ with $u-W$ is bounded above, then $u=W+c$ where $c$ is a negative constant as $u$ is a sign-changing solution. Observe that the function $f(t)=\left((t+c)^{+}\right)^{p} \in C^{1}(\mathbb{R})$ is non-increasing in a neighborhood of 0 and $W$ satisfies the equation

$$
\left\{\begin{array}{l}
-\Delta W=\left((W+c)^{+}\right)^{p} \text { in } \mathbb{R}^{N}, \\
W>0 \\
\lim _{x \longrightarrow \infty} W(x)=0 .
\end{array}\right.
$$

Therefore, $W$ is spherically symmetric a round some point $x_{0}$ and $\frac{\partial W}{\partial r}<0$, for all $r>0$ and as $\lim _{x \longrightarrow \infty} u(x)=c<0$ then $u^{+}$has a compact support $B_{R_{0}}\left(x_{0}\right), R_{0}>0$. Consequently $u$ satisfies

$$
\begin{cases}-\Delta u=u^{p} & \text { in } B_{R_{0}}\left(x_{0}\right) \\ u=0 & \text { on } \partial B_{R_{0}}\left(x_{0}\right) \\ u>0 & \end{cases}
$$

Taking into account that $p \geq \frac{N+2}{N-2}$, then by virtue of the Pohozaev identity, we derive that $u^{+}=0$, which is impossible since we assume that $u$ is a sign-changing solution.

Proof of (2). We will verify that if $u$ is a solution of $\left(E q \cdot S^{\prime}\right)$ or (1.6), then $u=0$. Two cases may occur.

Case 1. $\frac{N+2}{N-2}<p<p_{c}(N)$. Since $\gamma_{1}=(p-1) \frac{N}{2}-p \in\left[1, \gamma_{\max }\right)$, then for $\alpha>0$ small enough we have also $\gamma_{2}=(p-1) \frac{N}{2-\alpha}-p \in\left[1, \gamma_{\max }\right)$. Observe that $N-2 \frac{p+\gamma_{1}}{p-1}=0$ and $N-2 \frac{p+\gamma_{2}}{p-1}=\frac{-\alpha N}{2-\alpha}$. So, from (2.2) and (2.3) we deduce that

$$
\int_{R^{N}}|u|^{(p-1) \frac{N}{2}}<\infty,
$$

and

$$
\int_{\{R<|x|<4 R\}}|u|^{(p-1) \frac{N}{2-\alpha}} \leq C R^{\frac{-\alpha N}{2-\alpha}}, \forall R>R_{0} .
$$

Fix $\varepsilon>0$, then there exists $R_{1}=R_{\varepsilon}>R_{0}$ such that

$$
\int_{\{R<|x|\}}|u|^{(p-1) \frac{N}{2}}<\varepsilon, \forall R>R_{1} .
$$

Let $y \in \mathbb{R}^{N}$ such that $|y|=2 R$, then $B_{\frac{R}{2}}(y) \subset\{R<|x|<4 R\}$, and therefore

$$
\left\|u^{p-1}\right\|_{L^{2-\alpha}\left(B_{\frac{R}{2}}(y)\right)} \leq C R^{-\alpha}, \forall R>R_{1} .
$$

Following step 4 of the proof of Theorem 2 in [6] using Serrin's $L^{\infty}$-estimate, then from (3.12), (3.13) we can verify that $u$ satisfies

$$
|x|^{\frac{2}{p-1}} u(x) \rightarrow 0 \text { and }|x|^{1+\frac{2}{p-1}}|\nabla u(x)| \rightarrow 0 \text { as } x \rightarrow \infty .
$$


Therefore, similarly to step 5 of the proof of Theorem 2 in [6], we deduce that $u \equiv 0$.

Case 2. $1<p<\frac{N+2}{N-2}$. According to estimate (2.2) of Proposition 2.1 with $\gamma=1$, and as $N-2 \frac{p+1}{p-1}<0$ we may see that

$$
\int_{\mathbb{R}^{N}}|\nabla(u)|^{2}<\infty \text { and } \int_{\mathbb{R}^{N}}|u|^{p+1}<\infty
$$

Consequently, combining equation (1.6) with the Pohozaev identity we derive (see for instance [23, 10] for more details) that

$$
\int_{\mathbb{R}^{N}}|u|^{p+1}=\frac{2 N}{(N-2)(p+1)} \int_{\mathbb{R}^{N}}|u|^{p+1}
$$

and so $u \equiv 0$.

\section{Proof of Theorem 2.3.}

Proof of the "only if" part. Assume that $u_{n}$ is uniformly bounded, that is there exists $M_{0}>0$ such that $\left\|u_{n}\right\|_{L^{\infty}(\Omega)} \leq M_{0}, \forall n \in \mathbb{N}^{*}$. Since $f \in C^{1}(\mathbb{R})$, then there exists $M_{0}^{\prime}>0$ such that $\left\|f^{\prime}\left(u_{n}\right)\right\|_{L^{\infty}(\Omega)} \leq M_{0}^{\prime}$. Consider $0<\lambda_{1}<\lambda_{2} \ldots \leq \lambda_{k}$ the eigenvalues of the Neumann boundary value problem

$$
\begin{cases}-\Delta \varphi_{k}+\varphi_{k}=\lambda_{k} \varphi_{k} & \text { in } \Omega \\ \frac{\partial \varphi_{k}}{\partial \nu}=0 & \text { on } \partial \Omega\end{cases}
$$

where $\varphi_{k}$ is the corresponding eigenfunction of $\lambda_{k}$ satisfying

$$
\int_{\Omega} \varphi_{k}^{2}=1, \int_{\Omega} \nabla \varphi_{k} \nabla \varphi_{j}+\varphi_{k} \varphi_{j}=0, j \neq k \text { and } \int_{\Omega}\left|\nabla \varphi_{k}\right|^{2}+\varphi_{k}^{2}=\lambda_{k} \int_{\Omega} \varphi_{k}^{2}=\lambda_{k} .
$$

So $\left(\varphi_{k}\right)_{k \geq 1}$ constitute an orthogonal basis of $H^{1}(\Omega)$ and an Hilbert basis of $L^{2}(\Omega)$. Also $\lambda_{n} \rightarrow \infty$ as $n \rightarrow \infty$, therefore we can choose $n_{0} \in \mathbb{N}^{*}$ large enough such that $\lambda_{n_{0}}>M_{0}^{\prime}$. Set $E_{0}=<\varphi_{1}, \ldots, \varphi_{n_{0}}>$, then for every $h \in E_{0}^{\perp}$, we have $Q_{u_{n}}(h)=\int_{\Omega}|\nabla h|^{2}+h^{2}-\int_{\Omega} f^{\prime}\left(u_{n}\right) h^{2} \geq\left(\lambda_{n_{0}}-M_{0}^{\prime}\right) \int_{\Omega} h^{2}>0$, which implies that $i\left(u_{n}\right)$ is less than the dimension of $E_{0}$, and so the sequence of the Morse index $i\left(u_{n}\right)$ is bounded.

Proof of the " if" part. Blow-up analysis. Let $u_{n}$ be a sequence of solutions to (1.1). Suppose by contradiction that there exist a subsequence (which still denoted by $u_{n}$ ) and $i_{0} \in \mathbb{N}^{*}$ such that $i\left(u_{n}\right) \leq i_{0}$ but $\left\|u_{n}\right\|_{L^{\infty}(\Omega)} \rightarrow+\infty$.

Case 1. $p^{-}=p^{+}=p$. Set $M_{n}=\max _{\bar{\Omega}}\left|u_{n}(x)\right|:=\left|u_{n}\left(x_{n}\right)\right|, x_{n} \in \bar{\Omega}$. If $x_{n} \in \Omega$, set $d_{x_{n}}=\operatorname{dist}\left(x_{n}, \partial \Omega\right)$, as $\Omega$ is a bounded domain then according to the universal estimate established in [4], we may see that $d_{x_{n}}^{\frac{2}{p-1}} M_{n} \leq C\left(d_{x_{n}}^{\frac{2}{p-1}}+1\right) \leq C_{\Omega}$. Since we assume that $M_{n} \rightarrow+\infty$, then $d_{x_{n}}=\operatorname{dist}\left(x_{n}, \partial \Omega\right) \rightarrow 0$ and so $x_{n} \rightarrow x_{0} \in \partial \Omega$ up to subsequence denoted again $u_{n}$. By flattening the boundary through a local change of coordinate we may assume that near $x_{0}$ the boundary is contained in $\left\{x_{N}=0\right\}$ ( see the proof of theorem 3 in [22], precisely case 2.2 , page 1874, for more details). Using standard scaling and a blow-up 
argument as in [3], we obtain the following limiting equation 3

$$
\begin{cases}-\Delta u=\frac{1}{p}|u|^{p-1} u & \text { in }\left\{x_{N}>-d\right\} \\ \frac{\partial u(x)}{\partial x_{N}}=0 & \text { on }\left\{x_{N}=-d\right\} \\ d \geq 0,|u(0)|=1 & \text { and }|u(x)| \leq 1\end{cases}
$$

Also following [3, 4, 16] we can verify that $i(u)<\infty$. Set $x_{d}=(0,0, . ., d)$ and $u_{d, p}=p^{\frac{1}{p-1}} u\left(x-x_{d}\right)$, then $u_{d, p}$ satisfies $\left(E q . H^{\prime}\right)$. Therefore we reach a contradiction from Theorem 2.1 since we have $u_{d, p} \equiv 0$ but $\left|u_{d, p}\left(x_{d}\right)\right|=p^{\frac{1}{p-1}}$.

Case 2. $\frac{N+2}{N-2}<p^{-}<p^{+}<p_{c}(N)$. Set $M_{n}^{+}=\max _{\bar{\Omega}} u_{n}^{+}(x):=u_{n}\left(x_{n}^{+}\right)$and $M_{n}^{-}=\max _{\bar{\Omega}} u_{n}^{-}(x):=$ $-u_{n}\left(x_{n}^{-}\right), x_{n}^{+}, x_{n}^{-} \in \bar{\Omega}$. Without loss of generality we may assume that $x_{n}^{+}$(resp. $x_{n}^{-}$) converges to $\bar{x}^{+}$ $\left(\operatorname{resp} . \bar{x}^{-}\right) \in \bar{\Omega}$. Three subcases may occur:

Subcase $1 . \frac{M_{n}^{-}}{M_{n}^{+}} \geq C$ and $\frac{\left(M_{n}^{+}\right)^{p^{+}}}{\left(M_{n}^{-}\right)^{p^{-}}} \geq C$. We shall give special attention to subcase 1 since we normalize by $M_{n}^{+}$which is not necessarily an absolute maximum of $u_{n}$. Define

$$
\widetilde{u_{n}}(x):=\frac{u_{n}}{M_{n}^{+}}\left(x_{n}^{+}+\left(M_{n}^{+}\right)^{-\alpha} x\right), x \in \widetilde{\Omega_{n}}:=\left(M_{n}^{+}\right)^{\alpha}\left(\Omega-x_{n}^{+}\right), \text {with } \alpha=\frac{p^{+}-1}{2},
$$

and

$$
f_{n}(x):=\frac{1}{\left(M_{n}^{+}\right)^{p^{+}}} f\left(x_{n}^{+}+\left(M_{n}^{+}\right)^{-\alpha} x, M_{n}^{+} \widetilde{u_{n}}\right) .
$$

We have $\widetilde{u_{n}}(0)=1, \widetilde{u_{n}} \leq 1$ and $\widetilde{u_{n}}$ satisfies

$$
\begin{cases}-\Delta \widetilde{u_{n}}=f_{n}(x) & \text { in } \widetilde{\Omega_{n}}, \\ \frac{\partial \widetilde{u_{n}}}{\partial \nu}=0 & \text { on } \partial \widetilde{\Omega_{n}}\end{cases}
$$

From $\left(H_{1}\right)$ we have

$$
|f(x, s)| \leq C\left(1+\left(s^{-}\right)^{p^{-}}+\left(s^{+}\right)^{p^{+}}\right), \forall(x, s) \in \bar{\Omega} \times \mathbb{R} .
$$

Since we assume in this subcase that $\frac{\left(M_{n}^{+}\right)^{p^{+}}}{\left(M_{n}^{-}\right)^{p^{-}}} \geq C$, then

$$
\left|f_{n}(x)\right|=\frac{1}{\left(M_{n}^{+}\right)^{p^{+}}}\left|f\left(x_{n}+\left(M_{n}^{+}\right)^{-\alpha} x, M_{n}^{+} \widetilde{u_{n}}\right)\right| \leq C^{\prime} .
$$

Consequently, $\left\|f_{n}\right\|_{L^{\infty}\left(\widetilde{\Omega_{n}}\right)}$ is uniformly bounded. Note that we cannot apply here the universal estimate of [4] to exclude the limiting equation on the whole space because $f$ has no the same asymptotical behavior at $\pm \infty$. So, we have to discuss two cases:

- If $\left(M_{n}^{+}\right)^{\alpha} d_{n} \rightarrow+\infty$ then for any $R>0$ there exists $n_{0}$, such that $\forall n \geq n_{0}$, we have $B_{R} \subset \widetilde{\Omega_{n}}$. Recall now the following local $L^{p}$ - elliptic estimate: For $q>1$, we have

$$
\left\|\widetilde{u_{n}}\right\|_{W^{2, q}\left(B_{\frac{R}{2}}\right)} \leq C_{R}\left(\left\|f_{n}\right\|_{L^{q}\left(B_{R}\right)}+\left\|\widetilde{u_{n}}\right\|_{L^{q}\left(B_{R}\right)}\right) .
$$

We have $f_{n}$ is bounded $L^{q}\left(B_{R}\right)$ as it is bounded in $L^{\infty}\left(B_{R}\right)$. However, since we have only $\widetilde{u_{n}} \leq 1$, we cannot derive the boundedness of $\widetilde{u_{n}}$ in $L^{q}\left(B_{R}\right)$. Therefore we shall use Harnack's inequality combining 
with $L^{p}$ regularity theory to show that $\widetilde{u_{n}}$ is bounded in $L^{\infty}\left(B_{\frac{R}{2}}\right)$. Consider $v_{n}$ satisfying

$$
\left\{\begin{array}{ll}
-\Delta v_{n}=0 & \text { in } B_{R} \\
v_{n}=\widetilde{u_{n}} & \text { on } \partial B_{R} .
\end{array}\left(P^{\prime}\right)\right.
$$

Then $w_{n}:=\widetilde{u_{n}}-v_{n}$ verifies

$$
\begin{cases}-\Delta w_{n}=f_{n} & \text { in } B_{R}, \\ w_{n}=0 & \text { on } \partial B_{R} .\end{cases}
$$

Invoking $L^{p}$ - elliptic regularity, for $q>N$ we have

$$
\left\|w_{n}\right\|_{C^{1, \alpha}\left(B_{R}\right)} \leq C_{R}\left\|w_{n}\right\|_{W^{2, q}\left(B_{R}\right)} \leq C_{R}\left\|f_{n}\right\|_{L^{q}\left(B_{R}\right)} \leq C_{R}^{\prime}
$$

As $\widetilde{u_{n}} \leq 1$, then $\left(1-v_{n}\right)$ in a nonnegative harmonic function on $B_{R}$, then from Harnack's inequality, there holds

$$
\sup _{B_{\frac{R}{2}}}\left(1-v_{n}\right) \leq C_{R} \inf _{B_{\frac{R}{2}}}\left(1-v_{n}\right),
$$

which implies that $\inf _{B_{\frac{R}{2}}} v_{n} \geq 1-C_{R}+C_{R} \sup _{B_{\frac{R}{2}}} v_{n}$. Since $v_{n}(0)=\widetilde{u_{n}}(0)-w_{n}(0)$, it follows that $v_{n}(0) \geq$ $1-C_{R}$ and therefore $1-C_{R} C_{R}^{\prime} \leq v_{n}(x) \leq 1 ; \forall x \in B_{\frac{R}{2}}$. As $\widetilde{u_{n}}=w_{n}+v_{n}$, then

$$
\left\|\widetilde{u_{n}}\right\|_{L^{\infty}\left(B_{\frac{R}{2}}\right)} \leq C_{R}
$$

Consequently, $\widetilde{u_{n}}$ is bounded in $W^{2, q}\left(B_{\frac{R}{2}}\right), q>N$ for all $R>0$. From a standard diagonal subsequence

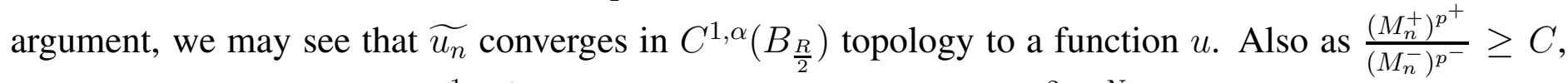
then $\left(H_{1}\right)$ implies that $f_{n} \rightarrow \frac{1}{p}\left(u^{+}\right)^{p}$ pointwise. In conclusion, $u \in C^{2}\left(\mathbb{R}^{N}\right)$ and satisfies

$$
\left\{\begin{array}{l}
-\Delta u=\frac{1}{p}\left(u^{+}\right)^{p^{+}} \quad \text { in } \mathbb{R}^{N} \\
u \text { is bounded above and } u(0)=1 .
\end{array}\right.
$$

Set $u_{p}(x)=p^{\frac{1}{p-1}} u(x)$, then $u_{p}$ is a finite Morse index solution of (1.8). So by virtue of Theorem 2.1 we deduce that $u=c \leq 0$. We reach a contradiction since $u_{p}(0)=p^{\frac{1}{p-1}}=1$.

- If $\left(M_{n}^{+}\right)^{\alpha} d_{n} \leq C$, we may assume that near $\bar{x}^{+}$, the boundary $\partial \Omega$ is contained in the plane $x^{N}=0$. There exists $\hbar$ a neighborhood of $\bar{x}^{+}$in $\mathbb{R}^{N}$ and $\omega: B_{1} \longrightarrow \hbar$ a $C^{1}$-diffeomorphism such that

$$
\omega\left(B_{1}^{+}\right)=\hbar \cap \Omega \text { and } \omega\left(B_{1}^{0}\right)=\hbar \cap \partial \Omega
$$

where

$$
\begin{aligned}
B_{1}^{+} & =\left\{x=\left(x^{\prime}, x_{N}\right) \in B_{1} ; x_{N}>0\right\}, \\
B_{1}^{0} & =\left\{x=\left(x^{\prime}, x_{N}\right) \in B_{1} ; x_{N}=0\right\} .
\end{aligned}
$$

Taking the change of variable $y=\omega^{-1}(x)$, we set $\widetilde{v}_{n}(y)=\frac{v_{n}}{M_{n}^{+}}\left(y_{n}^{+}+\left(M_{n}^{+}\right)^{-\alpha} y\right)$, with $v_{n}(y)=u_{n}(\omega(y))$ and $y_{n}^{+}=\omega^{-1}\left(x_{n}^{+}\right)$. This change of coordinates transforms the Laplacian operator into a general second 
order strongly elliptic operator. Moreover, up to subsequence we have $\left(y_{n}^{N} M_{n}^{+}\right)^{\alpha} \rightarrow d \geq 0$. Therefore, the blow-up analysis leads to the following limiting equation

$$
\left\{\begin{array}{l}
-\Delta u=\frac{1}{p}\left(u^{+}\right)^{p^{+}} \quad \text { in }\left\{x_{N}>-d\right\}, d \geq 0 \\
u(0)=1 \\
\frac{\partial u(x)}{\partial x_{N}}=0 \quad \text { on }\left\{x_{N}=-d\right\}, d \geq 0 \\
i(u)<\infty
\end{array}\right.
$$

As above, set $u_{d, p}=p^{\frac{1}{p-1}} u\left(x-x_{d}\right)$, we have $u_{d, p}$ satisfies $\left(E q . H^{\prime}\right)$ if $u$ is positive; or $(E q . H)$ if $u$ is sign-changing and from Theorem 2.1 we derive that $u \equiv c \leq 0$ which contradicts $u_{d, p}\left(x_{d}\right)=p^{\frac{1}{p-1}}$.

Subcase $2 . \frac{M_{n}^{-}}{M_{n}^{+}} \rightarrow 0$. We define

$$
\widetilde{u}_{n}(x)=\frac{u_{n}}{M_{n}^{+}}\left(x_{n}^{+}+\left(M_{n}^{+}\right)^{-\alpha} x\right), x \in \widetilde{\Omega_{n}}:=\left(M_{n}^{+}\right)^{\alpha}\left(\Omega-x_{n}^{+}\right), \text {with } \alpha=\frac{p^{+}-1}{2} .
$$

- If $\left(M_{n}^{+}\right)^{\alpha} d_{n} \rightarrow+\infty$, with $d_{n}=\operatorname{dist}\left(x_{n}^{+}, \partial \Omega\right)$. Hence, after blow up argument we obtain the limiting equation

$$
\left\{\begin{array}{l}
-\Delta u=\frac{1}{p} u^{p^{+}} \quad \text { in } \mathbb{R}^{N} \\
u(0)=1 \\
u>0 \\
i(u)<\infty
\end{array}\right.
$$

Apply Farina's result [6] we reach a contradiction.

- If $\left(M_{n}^{+}\right)^{\alpha} d_{n} \leq C$, similarly to case 1 , we derive the limiting equation

$$
\left\{\begin{array}{l}
-\Delta u=\frac{1}{p} u^{p^{+}} \quad \text { in }\left\{x_{N}>-d\right\}, d \geq 0, \\
u(0)=1 \\
u>0 \\
\frac{\partial u(x)}{\partial x_{N}}=0 \text { on }\left\{x_{N}=-d\right\}, d \geq 0 \\
i(u)<\infty .
\end{array}\right.
$$

As $u(0)=1$, a contradiction follows from (2) of Theorem 2.1.

Subcase 3. $\frac{M_{n}^{-}}{M_{n}^{+}} \geq C$ and $\frac{\left(M_{n}^{+}\right)^{p^{+}}}{\left(M_{n}^{-}\right)^{p^{-}}} \rightarrow 0$. Applying the blow-up argument around $x_{n}^{-}$where we normalize by $M_{n}^{-}$, we obtain a negative solution of the limiting equations

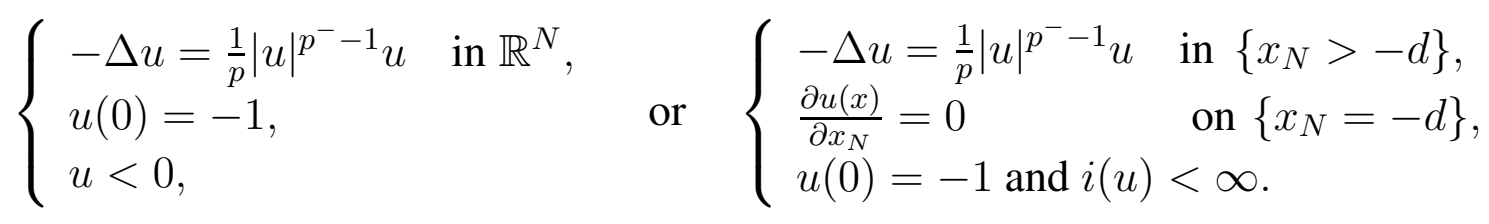

We derive respectively a contradiction from [6] and (2) of Theorem 2.1. This ends the proof of Theorem 2.3 .

\section{Acknowledgements}

The authors are greatly indebted to the deanship of Scientific Research at Northern Border University for its funding of the present work through the research project No.SCI-2018-3-9-F- 7931. 


\section{Bibliography}

[1] A. Ambrosetti and P. H. Rabinowitz, Dual variational methods in critial point theory and applications, J. Funct. Anal. 14 (1973), 369-381.

[2] A. Bahri and P. L. Lions, Solutions of superlinear elliptic equations and their Morse indices, Comm. Pure Appl. Math. XLV (1992), 1205-1215.

[3] A. Bahri and P. L. Lions, Morse index of some mini-max critical points, I : Application to multiplicity results, Comm. Pure Appl. Math. 41 (1988), 1027-1037.

[4] J. Davila, L. Dupaigne and A. Farina, Partial regularity of finite Morse index solutions to the Lane-Emden equation, J. Funct. Anal. 261 (2011), 218-232.

[5] D. G. de Figueiredo and J. Yang, On a semilinear elliptic problem without (PS) condition, J. Differntial Equations 187 (2003), 412-428.

[6] A. Farina, On the classification of solutions of the Lane-Emden equation on unbounded domains of $\mathbb{R}^{N}$, J. Math. Pures Appl. 87 (2007), 537-561.

[7] H. Hajlaoui, A. Harrabi and F. Mtiri, Morse indices of solutions for super-linear elliptic PDEs, Nonlinear Analysis 116 (2015), 180-192.

[8] H. Hajlaoui, A. Harrabi and D. Ye, On stable solutions of biharmonic problem with polynomial growth, Pacific Journal of Mathematics, 270 (2014), 79-93.

[9] M. K. Hamdani, A. Harrabi, $L^{\infty}$-norm Estimates of Weak Solutions via their Morse indices for the m-Laplacian Problems. Results in Mathematics, 74(1), p. 69 (2019).

[10] A. Harrabi, High-order Bahri-Lions Liouville type theorems, Annali di Matematica Pura and Applicata. (2019), DOIhttps://doi.org/10.1007/s1023.

[11] A. Harrabi, F. Mtiri and D. Ye, Explicit $L^{\infty}$-norm estimates via Morse index for the bi-harmonic and tri-harmonic semilinear problems, Manuscripta math. 159: (2019), 57-79.

[12] A. Harrabi and S. Rebhi, $L^{\infty}$-bounds for solutions of supercritical elliptic problems with finite Morse index, Adv.Nonlinear Stud. 10 (2010), 741-756.

[13] A. Harrabi, M. Ould Ahmedou, S. Rebhi and A. Selmi, A priori estimates for superlinear and subcritical elliptic equations: the Neumann boundary condition case. Manuscripta Math. 137 (2012), 525-544.

[14] A. Harrabi, B. Rahal, Liouville type theorems for elliptic equations in half-space with mixed boundary value conditions, Advances in Nonlinear Analysis, 8 (1) (2016), 193-202.

[15] A. Harrabi, S. Rebhi and A. Selmi, Solutions of superlinear equations and their Morse indices I, Duke. Math. J. 94 (1998), 141-157.

[16] A. Harrabi, S. Rebhi and A. Selmi, Solutions of superlinear equations and their Morse indices II, Duke. Math. J. 94 (1998), 159-179.

[17] D. D. Joseph and T. S. Lundgren, Quasilinear Dirichlet problems driven by positive sources, Arch. Rational Mech. Anal. 49 (1973), 241-269.

[18] D. Louis and A. Harrabi, The Lane-Emden equation in strips, Proceedings of the Royal Society of Edinburgh Section A: Mathematics 148 (1) (2018), 51-62.

[19] M. Ramos and P. Rodrigues On a fourth order superlinear elliptic problem, Electron. J. Diff. Eqns, Conf. 06 (2001), 243-255.

[20] M. Ramos, H. Tavares and W. Zou, A Bahri-Lions theorem revisted, Advances in Mathematics 222 (2009), 2173-2195 
[21] S. Rebhi, Characterization of solutions having finite Morse index for some nonlinear PDE with supercritical growth, Nonlinear Analysis T. M. A 74 (2011), 1182-1189.

[22] W. Reichel and T. weth, A priori bounds and a Liouville theorem on a half space for higher-order elliptic Dirichlet problems, Math. Z. 261 (2009), 805-827.

[23] A. Selmi, A. Harrabi and C. Zaidi, Nonexistence results on space and half space of $-\Delta u+\lambda u=|u|^{p-1} u$ via the Morse index, Accepted in CPAA.

[24] G. Wang and D. Ye, On a nonlinear elliptic equation arising in a free boundary problem, Math. Z. 244 (2003), 531-548.

[25] J. Yang, Existence of solutions of semilinear elliptic problem without (PS) condition, Proc. Amer. Math. Soc. 132(5) (2004), 1355-1366.

[26] X. F. Yang, Nodal Sets and Morse Indices of Solutions of Super-linear Elliptic PDE's, J. Funct. Anal. 160 (1998), 223-253. 